\title{
How much does the treatment of each major disease cost? A decomposition of Swiss National Health Accounts
}

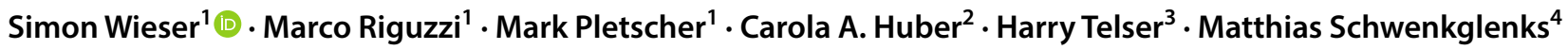

Received: 30 August 2017 / Accepted: 14 February 2018 / Published online: 22 February 2018

(c) The Author(s) 2018. This article is an open access publication

\begin{abstract}
In most countries, surprisingly little is known on how national healthcare spending is distributed across diseases. Singledisease cost-of-illness studies cover only a few of the diseases affecting a population and in some cases lead to untenably large estimates. The objective of this study was to decompose healthcare spending in 2011, according to Swiss National Health Accounts, into 21 collectively exhaustive and mutually exclusive major disease categories. Diseases were classified following the Global Burden of Disease Study. We first assigned the expenditures directly mapping from National Health Accounts to the 21 diseases. The remaining expenditures were assigned based on diagnostic codes and clues contained in a variety of microdata sources. Expenditures were dominated by non-communicable diseases with a share of 79.4\%. Cardiovascular diseases stood out with $15.6 \%$ of total spending, followed by musculoskeletal disorders (13.4\%), and mental and substance use disorders (10.6\%). Neoplasms (6.0\% of the total) ranked only sixth, although they are the leading cause of premature death in Switzerland. These results may be useful for the design of health policies, as they illustrate how healthcare spending is influenced by the epidemiological transition and increasing life expectancy. They also provide a plausibility check for single cost-of-illness studies. Our study may serve as a starting point for further research on the drivers of the constant growth of healthcare spending.
\end{abstract}

Keywords Healthcare expenditures $\cdot$ Cost-of-illness $\cdot$ Healthcare costs $\cdot$ National Health Accounts $\cdot$ Switzerland . Decomposition by diseases

JEL Classification I10 Health General · I11 Analysis of Health Care Markets

Electronic supplementary material The online version of this article (doi:https://doi.org/10.1007/s10198-018-0963-5) contains supplementary material, which is available to authorized users.

Simon Wieser

simon.wieser@zhaw.ch

Marco Riguzzi

marco.riguzzi@zhaw.ch

Mark Pletscher

mark.pletscher@roche.com

Carola A. Huber

carola.huber@helsana.ch

Harry Telser

harry.telser@polynomics.ch

Matthias Schwenkglenks

matthias.schwenkglenks@uzh.ch

\section{Introduction}

Although it is quite well known how healthcare spending is financed and what kind of services are bought, surprisingly little is known on how much is spent on each major disease.

1 Winterthur Institute of Health Economics, Zurich University of Applied Sciences, Gertrudstrasse 15, 8401 Winterthur, Switzerland

2 Department of Health Sciences, Helsana Insurance Group, Zurich, Switzerland

3 Polynomics, Olten, Switzerland

4 Epidemiology, Biostatistics and Prevention Institute, University of Zurich, Zurich, Switzerland 
Policy interventions that aim at improving population health require a thorough understanding of the importance of single diseases and the evolution of disease-specific expenditures over time. Disease-specific expenditures are important because they are likely to change with changes in access to care, medical progress, and increasing life expectancy.

A growing number of cost-of-illness studies have assessed the costs of single diseases in a variety of countries [1]. These studies usually cover only a fraction of the diseases affecting a country's population, and they are often criticized for overestimating the true costs of diseases [2, 3]. This overestimation may be due to methodological difficulties in isolating the expenditures for a specific disease in patients affected by multiple conditions. It may also be the consequence of an overestimation bias when researchers highlight the economic impact of the disease under study.

The aim of the present study was to decompose total healthcare spending in Switzerland in 2011 by disease and by other reasons for healthcare use. We applied a general cost-of-illness approach [3] assigning National Health Accounts (NHA) healthcare expenditures to a collectively exhaustive and mutually exclusive set of diseases, thus avoiding double counting. NHA are published by the national statistical offices of many high-income countries and by the Swiss Federal Office of Statistics for Switzerland [4]. NHA assess the current level of healthcare expenditures and decompose them by type of financing source, financing regimen, service provider and service.

The System of Health Accounts 2011 that was coordinated by OECD, Eurostat and WHO suggests a comprehensive breakdown by disease [5]. A decomposition by diseases is, however, not part of the currently applied NHA standards. Nonetheless, healthcare expenditures have been partially decomposed by diseases for number of OECD countries including Australia, Canada, Korea, Japan and several European countries [6-8]. Furthermore, expenditures in numerous low- and middle-income countries have been decomposed by the diseases of their key areas' health burden, such as communicable diseases and conditions related to maternal and child health [9]. However, the most comprehensive efforts to decompose spending by diseases have been carried in the USA. While some studies have focused on decomposing US healthcare spending by diseases $[10,11]$, others have focused on the impact on spending of potential drivers, such as changes in cost per treated case or changes in treated prevalence [12-17]. To the best of our knowledge, the only study decomposing total spending by the same collectively exhaustive set of disease classifications was the study by Dieleman and coauthors [11], which decomposed US personal healthcare spending (corresponding to $85 \%$ of the US's NHA) into 155 diseases.

Our study may be interesting for health economists and public health researchers for a number of reasons: first, we combine a top-down approach, based on the disease information already contained in NHA, with a bottom-up approach based on patient-level microdata from a variety of sources. We perform a more detailed attribution of outpatient spending than most previous studies. Our approach also differs to some extent from the one followed by Dieleman et al. [11] and may be applied to countries with less rich data on healthcare expenditures than the USA. Second, Swiss NHA are relatively complete and detailed in comparison with those of many other high-income countries [18]. Our study illustrates how this multidimensional measurement of healthcare expenditures can serve as a starting point for further enquiries into the drivers of healthcare spending. Third, our study illustrates how expenditures are allocated across diseases in a country with high healthcare spending, universal access to high-quality care and very little rationing. Switzerland has one of the most expensive and generous healthcare systems with universal coverage and virtually no waiting time [19]. Per capita spending for health in Switzerland is among the highest in the world (USD 9813 in 2015) and at a similar level as in the USA (USD 9892 in 2016) [20]. ${ }^{1}$ Even the purchasing power adjusted level of spending in Switzerland is approximately $30 \%$ higher than in European countries with similar healthcare systems such as Germany or the Netherlands [20]. Life expectancy is one of the highest in the world [22]; surveys report very high ratings of self-assessed health, and overall satisfaction with healthcare services is high [19].

\section{Data and methods}

We decomposed 2011 Swiss NHA healthcare expenditures by 21 major diseases. We first defined a collectively exhaustive and mutually exclusive set of 21 diseases and 18 types of healthcare services and created a service-disease grid (described more thoroughly below) representing the overall envelope for the decomposition. Collectively, the cells of this grid aggregate to be $100 \%$ of NHA expenditures in Switzerland in 2011. Expenditures for each cell were estimated by either a top-down or a bottom-up approach. The top-down approach estimated expenditures based on the information already contained in the NHA. The bottom-up approach assigned expenditures that could not be assigned by the top-down approach based on the analysis of microdata on healthcare utilization. These microdata contained

\footnotetext{
1 Per capita spending was 9666 Swiss francs (CHF) in 2016 [20] with an exchange rate of $0.985 \mathrm{CHF} / \mathrm{USD}$ [21]. The share of healthcare spending in GDP was lower in Switzerland than in the US (12.4 vs. $17.2 \%$ ) [20] due to higher per capita income in Switzerland.
} 
Table 1 Classification by 21 diseases and other reasons for health service use Source: own adaptation of Global Burden of Disease Study (GBD) classification [23], with diseases ordered according to GBD

\begin{tabular}{|c|c|c|}
\hline 21 Major diseases & Example & Disease group on level 1 in GBD \\
\hline Communicable diseases & Lower respiratory infection & \multirow{3}{*}{$\begin{array}{l}\text { Group 1: diseases dominating the } \\
\text { burden of disease in low-income } \\
\text { countries }\end{array}$} \\
\hline Maternal and neonatal disorders & Neonatal preterm complication & \\
\hline Nutritional deficiencies & Iron-deficiency anemia & \\
\hline Neoplasms & Lung cancer & \multirow[t]{15}{*}{ Group 2: non-communicable diseases } \\
\hline Cardiovascular diseases & Ischemic heart disease & \\
\hline Chronic respiratory diseases & Asthma & \\
\hline Cirrhosis and other chronic liver diseases & Cirrhosis & \\
\hline Digestive diseases & Gastritis & \\
\hline Neurological disorders (without dementia) & Multiple sclerosis & \\
\hline Dementia & Alzheimer's disease & \\
\hline Mental and substance use disorders & Depression & \\
\hline $\begin{array}{l}\text { Urogenital, blood, and endocrine diseases (without } \\
\text { diabetes) }\end{array}$ & Chronic kidney disease & \\
\hline Diabetes & Diabetes mellitus & \\
\hline Musculoskeletal disorders & Low back pain & \\
\hline Congenital anomalies & Congenital heart anomaly & \\
\hline Skin diseases & Acne & \\
\hline Sense organ diseases & Hearing loss & \\
\hline Oral disorders & Periodontal disease & \\
\hline Injuries & Road injuries & Group 3: injuries \\
\hline Well care & Pregnancy and birth without complications & Not included in GBD classification \\
\hline Prevention & Accident prevention campaign & \\
\hline
\end{tabular}

additional information on both the types of diseases affecting individual patients and on related spending.

\section{Classification of diseases and healthcare services}

The first step was the identification of meaningful and appropriate categories of diseases and healthcare services at a suitable degree of granularity. A higher degree of granularity in the decomposition would not only allow for determining the spending for major disease groups, such as cardiovascular diseases, but also would allow for more specific sub-diseases, such as ischemic heart disease. However, the details of the diagnostic information in the used data strongly limited the possible granularity of the decomposition. Only one data source included ICD-10 codes, and we had only limited access to patient-level data with partial diagnostic information such as health insurance claims data. We thus chose the maximum achievable degree of granularity under the constraints of the available data and the financial resources for this study.

We adapted the disease classification used in the Global Burden of Disease Study (GBD) 2010 [23]. This study comprises three hierarchical classification levels, with 3 disease groups at level 1,21 disease groups at level 2, and 291 diseases and injuries at level 3. The use of the GBD classification has several advantages: first, it represents a mutually exclusive and collectively exhaustive classification of all diseases and injuries. Second, it is used by the GBDProject to assess the country-specific incidence and prevalence of diseases as well as their health burdens in terms of disability-adjusted life years (DALYs). The GBD classification thus allowed us to compare the healthcare spending for the treatment of diseases to the health burden in terms of DALYs of the same diseases. Third, the GBD classification was also used in a recent decomposition of US healthcare spending by disease [11], and the use of the same system allowed a comparison of the results.

We employed a modified version of the GBD level 2 classification (Table 1). The modifications included the addition of six disease categories within the non-communicable diseases and the combination of all communicable diseases into one category. We also added two additional categories for well care and prevention, which are not included in the GBD classification because they do not represent a disease burden. Well care includes pregnancy and birth without substantial complications and other non-disease treatments by healthcare providers such as family planning or non-reconstructive cosmetic procedures. Prevention includes health promotion and disease and injury prevention programs financed by public and private organizations. It also includes disease 
Table 2 Classification of healthcare services Source: own elaboration based on [4]

\begin{tabular}{|c|c|c|}
\hline 6 Main categories & 18 Sub-categories & Notes \\
\hline \multirow[t]{4}{*}{ Inpatient care ${ }^{\mathrm{a}}$} & Acute inpatient care & Acute care in hospitals and psychiatric clinics \\
\hline & Inpatient rehabilitation & Rehabilitation in rehabilitation clinics \\
\hline & Inpatient long-term care & Mainly nursing home care for the elderly \\
\hline & Other inpatient long-term & Care in specialized institutions for disabled patients ${ }^{b}$ \\
\hline \multirow[t]{7}{*}{ Outpatient care } & Outpatient physicians & Primary care physicians and specialists \\
\hline & Outpatient hospital & Care for patients without overnight stay \\
\hline & Outpatient dental care & Care and prevention by dentists \\
\hline & Outpatient physiotherapy & \\
\hline & Outpatient psychotherapy & \\
\hline & Outpatient nursing & Outpatient care for mainly elderly patients \\
\hline & Other paramedic services & Including alternative medicine \\
\hline \multirow[t]{2}{*}{ Health products } & $\begin{array}{l}\text { Drugs provided by physicians }{ }^{\mathrm{c}} \text { and } \\
\text { pharmacies }\end{array}$ & Prescription drugs, drugs provided by hospitals not included \\
\hline & Medical devices & Devices provided directly to patients \\
\hline \multirow[t]{3}{*}{ Other outpatient services } & Clinical laboratory & Only centralized laboratories outside hospitals \\
\hline & Radiology & Only specialists and specialized outpatient centers \\
\hline & Ambulance and rescue & \\
\hline Prevention & Prevention & Tertiary prevention by government agencies \\
\hline Administration & Administration & Public and private health insurers \\
\hline
\end{tabular}

${ }^{a}$ Expenditures include hoteling services for accommodations and food

${ }^{\mathrm{b}}$ Mainly disabilities due to mental disorders, congenital birth defects and sense organ diseases

${ }^{\mathrm{c}}$ Physicians may directly sell drugs to their patients in some Swiss cantons

prevention services delivered by healthcare providers such as vaccinations and screening examinations. Prevention spending is reported as a separate category because it would be very difficult to assign the spending for broad health promotion to single diseases. Furthermore, Larg and Moss [24] have suggested separately reporting prevention spending in cost-of-illness studies. Table 1 gives an overview of the 21 diseases and other reasons for health service use categories used in this study. For simplicity, we will refer to them as diseases emphasized in italics in the remainder of this text.

Healthcare services were classified using a slightly simplified version of the Swiss NHA from the type of service perspective (Table 2) [4]. ${ }^{2}$ The original type of service classification consisted of six main categories with 29 sub-categories. We omitted the sub-categories within prevention and administration and merged expenditures for drugs provided by pharmacies and by physicians into one category. Table 2 illustrates the resulting collectively exhaustive and mutually

\footnotetext{
2 Swiss NHA decompose health expenditures from four perspectives: (1) financing source, (2) financing regimen, (3) type of service provider, (4) type of service. In April 2017, the Swiss Federal Office of Statistics published a new version of NHA with a revised classification within these perspectives [13]. Our study is based on the previously published version.
}

exclusive classification of healthcare services with six main categories and 18 sub-categories.

The combination of 18 service categories and 21 disease categories resulted in a matrix of 378 service-disease cells. We pre-specified the row totals as the total spending for single healthcare services according to NHA, which constitute a constraint to the decomposition. The column totals per disease were calculated after the assignment of service-specific NHA expenditures to different diseases. They represent the main result of our study.

\section{Assigning expenditures to service-disease cells}

We used either a top-down or a bottom-up approach to estimate healthcare expenditures of each single service-disease cell. A number of cells were predefined as empty for certain diseases that should never require the use of certain healthcare services. Examples included prevention and well care, which should never require long-term nursing home care, or dental care, which should not be used for the treatment of cardiovascular or urogenital diseases.

\section{Top-down cells}

The top-down approach made use of the available information on disease-specific expenditures in the different 
dimensions of NHA. In the service-type dimension, the expenditures for psychotherapy and dental care were directly assigned to mental and substance use disorders and oral disorders. In the provider-type dimension, the expenditures for psychiatric hospitals were assigned to mental and substance use disorders and dementia. The Swiss funding mechanisms further allowed for assigning expenditures by accident insurance companies that cover all residents in paid jobs to injuries, and assigning expenditures by the mandatory disability insurance that covers healthcare expenditures due to congenital birth defects until the age of 20 years to congenital anomalies.

All public health spending for prevention was assigned to the service-disease cell prevention-prevention.

\section{Bottom-up cells}

The bottom-up approach used administrative data, insurance claims data, household survey data and physicians' billing data to assign expenditures to the service-disease cells that could not be assigned by the top-down approach. We applied a hierarchical procedure based on the following principles:

1. Single service-disease cells were filled with information from a unique data source, except for the cells that could only partially be assigned with the top-down approach.

2. We started with the data of the highest quality and broadest coverage, and then gradually passed to less ideal data sources. For example, we used an inpatient episode registry instead of health insurance claims data to assign acute inpatient care expenditures to diseases, because the inpatient episode registry had a higher coverage and more accurate diagnostic information. Conversely, we then used health insurance claims data as the preferred data source when assigning expenditures for hospital outpatient services to diseases.

The following section gives an overview of the procedures that were followed in assigning expenditures to diseases (see appendix for more details).

\section{Inpatient care}

Expenditures for inpatients in acute care hospitals, psychiatric clinics, rehabilitation clinics and nursing homes were assigned based on their main ICD-10 diagnoses coded in the Medical Statistic of Hospitals (MS). The MS is a registry of inpatient episodes by the Swiss Federal Office of Statistics (FOS). It covers all inpatient stays uniquely identifying patients across multiple stays in different hospitals. The main diagnosis coded in the MS was particularly well suited for our analysis, as it corresponds to the diagnosis responsible for most of the resources used during an inpatient stay [25].
We used the 2011 MS containing 1,363,697 inpatient stay records (98.3\% reported a diagnosis).

The procedures used to assign expenditures to single diseases differed between inpatient care categories, because information on expenditures and diagnoses also differed between them.

Expenditures for acute care hospitals were assigned based on the main diagnosis and the AP-DRG ${ }^{3}$ cost weights assigned to each hospital stay in the MS. These expenditures were calculated as a product of number of stays assigned to a disease and the corresponding AP-DRG cost weights in 2011 [26]. AP-DRG cost weights were missing for a relatively small number of stays (1.4\%). Stays in psychiatric hospitals or psychotherapeutic wards of acute care hospitals were assigned the average AP-DRG cost weight of the other stays in these wards. We then upscaled single acute care disease cells to match the NHA total for inpatient acute care by distributing residual expenditures proportionally to previously assigned acute care expenditures. This upscaling covered expenditure for yet unassigned stays and hospital investment expenditures not included in the AP-DRG cost weights, as well as additional charges for patients with private supplementary health insurance. ${ }^{4}$

The proportion of total NHA expenditures for inpatient care by psychiatric hospitals was calculated by subtracting the expenditures for outpatient services from the total expenditures for these hospitals. The resulting inpatient care expenditures for these hospitals were then assigned to mental and substance use disorders and dementia proportionally to the total number of treatments days with these diagnoses in the MS.

The coding quality of MS records of rehabilitation clinics was often poor or missing. However, many stays in these clinics were preceded by treatments in acute care hospitals (more than $61 \%$ in the preceding 3-week period). We thus used the diseases coded as a patient's main diagnosis in acute care hospitals to proportionally assign all rehabilitation clinic stays to disease groups. Expenditures for inpatient rehabilitation were calculated by multiplying the average daily expenditures for rehabilitation clinics with the total number of treatment days in each disease group.

As we found no data directly assessing the diagnoses leading to long-term care in nursing homes, we used the information included in the MS to assign these expenditures. We assessed the diagnoses of patients who were first referred to a nursing home after an inpatient stay in an acute

\footnotetext{
3 AP-DRG was a prospective payment system (PPS) for acute care hospital stays used in the western and central cantons of Switzerland until 2011. In 2012, the new national SwissDRG PPS replaced it [9].

${ }^{4}$ Private supplementary health insurance covers higher than standard hotel services and treatment by a head physician.
} 
care hospital. We did not assess the diagnoses of patients admitted to acute care from a nursing home, because these diagnoses might not be the cause of the first nursing home admission. As most patients in nursing homes were affected by multiple diseases, we identified the main diagnosis and the first two secondary diagnoses coded during the acute care episode before the first admission to the nursing home. All nursing home expenditures were then assigned proportionally to the number of diagnoses assigned to each of the 21 diseases.

\section{Outpatient services and healthcare products}

Expenditures for outpatient services and health products were assigned using a bottom-up approach based on microdata.

Attribution based on claims data Some part of national expenditures for outpatient physicians and hospital care could be assigned to single service-disease cells based on claims data provided by the Helsana health insurance group. With over a million insured individuals, Helsana was one of the largest providers of mandatory health insurance in Switzerland and held a market share of $14 \%$ in 2011. Pharmaceutical cost groups were used to identify seven noncommunicable diseases following the procedure described in Huber et al. [27]. The incremental expenditures associated with the identified diseases were estimated with a negative binomial regression with interaction terms. Total expenditures for each service-disease correspond to the product of incremental expenditures for a disease multiplied by its prevalence in the Helsana insurance population and then extrapolated to the overall population (see appendix for more details).

Attribution based on the Swiss Health Survey The 2012 Swiss Health Survey (SHS) [28] allowed for assigning the expenditures for a number of outpatient service cells including outpatient physician visits, physiotherapy, outpatient nursing and other outpatient services. The SHS is a representative survey of the Swiss population aged 15 years and older living in private households. It assesses the service utilization of respondents with questions about the number of consultations of different healthcare providers in the previous 12 months. Respondents also reported on the diseases affecting them. We calculated disease-specific spending by combining the information on service use and diseases affecting the respondents (see appendix for more details).

Attribution-based market research data We used market research data provided by IMS Health to assign the drug expenditures for a number of diseases. IMS Health provided contributions of these diseases to overall drug spend- ing, based on information from pharmacies, physicians, drugstores and hospitals in 2011. Diseases were classified according to Anatomical Therapeutic Chemical Classification (ATC) codes that map drugs to different disease groups. Due to budget restrictions, we could obtain these drug expenditure data just for ten diseases (see appendix for more details).

Attribution based on Medical Statistic of Hospitals The MS holds information on whether a patient was admitted to inpatient care by ambulance. We used this information to assess the number of inpatient stays with ambulance admission in the 21 major disease categories. Overall spending for transport and rescue was then assigned to disease categories proportionally to the number of ambulance admissions.

Attribution based on physicians' billing data The NewIndex physicians' billing database allowed us to assign expenditures to a number of additional service-disease cells. The database holds information on services and products, including drugs and laboratory tests, which were prescribed and provided in outpatient physician practices.

Expenditures could be mapped to diseases based on three types of information shown on physicians' invoices. (1) The physicians' specialization: expenditures related to a consultation with an oncologist were, for example, assigned to neoplasms. (2) The type of insurance: expenditures covered by accident insurance related, for example, to injuries. (3) The Tessiner Code: a minimalist disease classification based on relatively broad diagnostic categories (such as diabetes) and localizations of the body parts affected (such as spine).

Attribution to remaining service-disease cells At this point of our procedure, a number of service-disease cells were still empty. Some of these cells were left empty while the remainder could be assigned to some of the yet unassigned spending.

Cells could be left empty for the following three reasons: (1) The service-disease cell was empty by definition, such as inpatient rehabilitation for the disease category prevention or the service dental care for dementia; (2) the row total of a service category had already been assigned to other disease cells in this row, and this was the case for other long-term care expenditures assigned entirely to five diseases; (3) there was a lack of diagnostic information in the microdata used for outpatient service attribution, such as for nutritional deficiencies and for cirrhosis or for the healthcare service category of radiology.

Regarding the service-disease cells that were assigned as part of the not yet assigned expenditures, we proceeded as follows: (1) missing expenditures for outpatient physicians' service-disease cells were assigned in proportion to the share of inpatient acute care expenditures for these 
diseases; (2) missing expenditures for other outpatient service-disease cells were assigned in proportion to outpatient physician services; (3) the administrative expenditures of health insurance companies were distributed in proportion to the total inpatient and outpatient cost already assigned to a disease.

\section{Results}

We decomposed overall Swiss healthcare expenditures by disease, with similar results so far available only for the USA [11]. The decomposition made use of diagnostic codes and clues contained in a multitude of data sources on healthcare expenditure and service use in Switzerland. We were able to assign the majority of spending to diseases.

\section{Expenditures attributed with top-down and bottom-up approaches}

The Swiss NHA reported healthcare spending of $64.6 \mathrm{bn}$ Swiss francs (CHF) ${ }^{5}$ in 2011. Table 3 illustrates the assignment of these expenditures to the predefined 378-cell service-disease grid. A total of 75 cells were predefined as empty because the type of healthcare service did not fit with the type of disease. The top-down approach allowed for assigning $15.4 \%$ of total spending to 23 service-disease cells (mainly for mental and substance use disorders, congenital birth defects, injuries, and oral diseases).

The bottom-up approach assigned $75.2 \%$ of total spending to 170 service-disease cells based on the different data sources used:

- 38.6\% based on MS hospital registry data (79 servicedisease cells for different types of inpatient care and ambulance services),

- $14.0 \%$ based on Helsana health insurance claims data (14 cells for physician and hospital services for selected non-communicable diseases),

- $7.9 \%$ based on IMS market research data (13 cells for drugs),

- $5.5 \%$ based on Swiss Health Survey data (14 cells, mainly for outpatient service categories other than physicians and hospital services),

- $5.2 \%$ based on NewIndex physicians' billing data (31 cells, mainly physician services not assigned with Helsana data, drugs not assigned with IMS data and laboratory analysis across all disease categories).

\footnotetext{
5 Average exchange rates in 2011 were $0.811 \mathrm{EUR} / \mathrm{CHF}$ and 1.127 USD/CHF [21].
}

- $4.0 \%$ based on the main type of disability affecting the patients living in other long-term care institutions (4 cells). The remaining 15 cells for other long-term care remained empty.

At this stage of the procedure, $9.4 \%$ of total spending remained to be assigned. 5.5\% was attributed to 98 empty service-disease cells mainly regarding outpatient physician services, radiology and medical devices. The remaining $4.0 \%$ of spending comprised 18 health insurance administration cells, which were assigned proportionally to health service spending for diseases.

\section{Decomposition of expenditures by disease}

The main result of our study is the decomposition of the 2011 NHA expenditures by 21 major diseases. Table 4 presents these results in the 378 cell service-disease grid. The first three rows of the table show the total spending for each disease category in Swiss francs (CHF), the contribution of the disease to overall spending and the ranking of the diseases by share of expenditures. Figure 1 gives a visual overview of the relative contribution of diseases and disease groups to overall spending. The main results are summarized as follows:

- Expenditures were dominated by non-communicable diseases (79.4\%), followed by injuries (8.1\%), communicable, maternal and nutritional diseases $(6.4 \%)$, prevention (4.0\%), and well care (2.4\%).

- Cardiovascular diseases stood out as the most expensive disease category ( $15.6 \%$ of total spending), followed by musculoskeletal disorders (13.4\%), mental and substance use disorders (10.6\%), injuries (8.1\%), and urogenital, blood and endocrine diseases (6.8\%).

- Surprisingly, neoplasms ranked only sixth among the diseases with the highest expenditures although neoplasms were a leading cause of premature death in Switzerland with $35.2 \%$ of years of life lost due to premature death in 2010 [29].

- Prevention and well care, the two healthcare expenditure categories not related to the treatment of diseases, were responsible for $6.6 \%$ of spending $(4.0 \%$ for prevention and $2.4 \%$ for well care).

- Six diseases accounted for less than $2.0 \%$ of healthcare spending. Surprisingly, they also included dementia (1.7\% of total spending) and diabetes (1.5\% of total), two chronic non-communicable diseases that are often referred to as important drivers for increasing healthcare spending [30-32].

The relative importance of various service categories differed considerably between diseases (Fig. 2). The proportion 
Table 3 Overview of sources used to attribute expenditures in the service-disease grid

\begin{tabular}{|c|c|c|c|c|c|c|c|c|c|c|c|c|c|c|c|c|c|c|c|c|c|}
\hline & 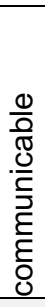 & 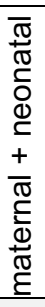 & 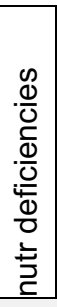 & 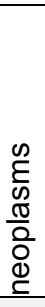 & 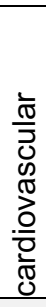 & 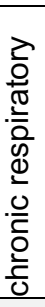 & $\begin{array}{l}\frac{0}{0} \\
\text { 은 } \\
\frac{c}{2} \\
\frac{1}{0}\end{array}$ & 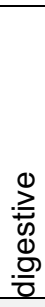 & 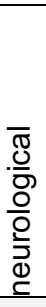 & 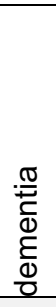 & 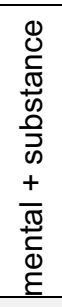 & 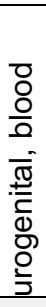 & $\begin{array}{l}\mathscr{\infty} \\
\frac{1}{1} \\
\frac{0}{0} \\
\frac{0}{0}\end{array}$ & 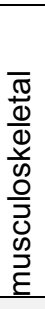 & 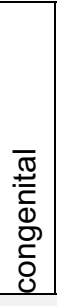 & $\frac{\frac{5}{v}}{\omega}$ & 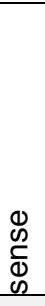 & $\begin{array}{l}\overline{0} \\
\text { 0 }\end{array}$ & 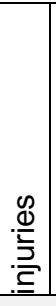 & $\begin{array}{l}\frac{\mathscr{d}}{\bar{J}} \\
\overline{\bar{O}} \\
\end{array}$ & $\begin{array}{l}\text { 을 } \\
\frac{c}{1} \\
\frac{1}{d} \\
\frac{1}{2}\end{array}$ \\
\hline \multicolumn{22}{|l|}{ Inpatient care } \\
\hline \multirow{4}{*}{$\begin{array}{l}\text { Acute care } \\
\text { Rehabilitation } \\
\text { Long-term nursing } \\
\text { Other long-term }\end{array}$} & $b$ & $b$ & $b$ & $b$ & $b$ & $b$ & $b$ & $b$ & $b$ & $a b$ & $a b c$ & $b$ & $b$ & $b$ & $a b$ & $b$ & $b$ & $b$ & $b$ & $b$ & v \\
\hline & $d$ & $d$ & $d$ & $d$ & $d$ & $d$ & $d$ & $d$ & $d$ & $d$ & $d$ & $d$ & $d$ & $d$ & $d$ & $d$ & $d$ & $d$ & $d$ & v & v \\
\hline & $b$ & $b$ & $b$ & $b$ & $b$ & $b$ & $b$ & $b$ & $b$ & $b$ & $b$ & $b$ & $b$ & $b$ & $b$ & $b$ & $b$ & $b$ & $b$ & V & v \\
\hline & w & w & w & w & w & w & W & w & w & w & e & w & w & e & e & w & e & w & w & V & v \\
\hline \multicolumn{22}{|l|}{ Outpatient care } \\
\hline Physicians & $g$ & g & $x$ & $f$ & $f$ & $f$ & $x$ & $g$ & $g$ & f & $f$ & g & $f$ & $f$ & a & $g$ & $g$ & $g$ & a & $h$ & $\mathrm{~h}$ \\
\hline Hospitals & $x$ & $x$ & $x$ & $f$ & f & $f$ & $x$ & $x$ & $x$ & f & f & $x$ & f & $f$ & $x$ & $x$ & $x$ & $x$ & a & $x$ & $x$ \\
\hline Dental care & v & v & V & v & V & v & V & v & v & v & v & V & v & v & v & V & v & a & v & V & v \\
\hline Physiotherapy & $x$ & $x$ & $x$ & $x$ & $\mathrm{~h}$ & $x$ & $x$ & $x$ & $x$ & $x$ & $h$ & $x$ & $x$ & $\mathrm{~h}$ & a & $x$ & $x$ & $x$ & a & $x$ & v \\
\hline Psychotherapy & V & v & V & v & V & v & V & v & v & v & a & V & v & v & v & V & v & v & v & V & v \\
\hline Outpatient nursing & $x$ & $x$ & $x$ & $\mathrm{~h}$ & $\mathrm{~h}$ & $x$ & $x$ & $x$ & $x$ & $x$ & $\mathrm{~h}$ & $x$ & $\mathrm{~h}$ & $\mathrm{~h}$ & a & $x$ & $x$ & $x$ & a & V & v \\
\hline Other outpatient care & $x$ & $x$ & $x$ & h & $x$ & h & $x$ & $\mathrm{x}$ & $x$ & $x$ & $\mathrm{~h}$ & $x$ & $x$ & h & a & $x$ & $x$ & $x$ & a & V & v \\
\hline \multicolumn{22}{|l|}{ Other services } \\
\hline \multirow{3}{*}{$\begin{array}{l}\text { Laboratory analysis } \\
\text { Radiology } \\
\text { Transport and rescue }\end{array}$} & $g$ & $g$ & $x$ & g & $g$ & $g$ & $x$ & g & g & $x$ & $g$ & $g$ & $g$ & g & a & $g$ & g & $g$ & ag & $g$ & g \\
\hline & $x$ & $x$ & $x$ & $x$ & $x$ & $x$ & $x$ & $x$ & $x$ & $x$ & $x$ & $\mathrm{x}$ & $x$ & $x$ & $x$ & $x$ & $x$ & $x$ & a & $x$ & $x$ \\
\hline & $i$ & $i$ & $i$ & $i$ & $i$ & $i$ & $i$ & $i$ & $i$ & $i$ & $i$ & $i$ & $i$ & $i$ & $i$ & $i$ & $i$ & $i$ & ai & $i$ & v \\
\hline \multicolumn{22}{|l|}{ Sales of health goods } \\
\hline \multirow{2}{*}{$\begin{array}{l}\text { Drugs } \\
\text { Medical devices }\end{array}$} & j & $g$ & $x$ & $j$ & $j$ & $j$ & $x$ & $j$ & $j$ & j & $j$ & $j$ & $j$ & j & $j$ & $j$ & g & $g$ & $g$ & $g$ & g \\
\hline & $x$ & $x$ & $x$ & $x$ & $x$ & $x$ & $x$ & $x$ & $x$ & $x$ & $x$ & $x$ & $x$ & $x$ & a & $x$ & $x$ & $x$ & $a$ & $x$ & V \\
\hline \multirow{2}{*}{$\begin{array}{l}\text { Prevention } \\
\text { Administration }\end{array}$} & V & V & V & V & V & V & V & V & V & V & V & V & V & V & V & V & V & V & V & V & a \\
\hline & $y$ & $y$ & $y$ & $y$ & $y$ & $y$ & $y$ & $y$ & $y$ & $y$ & $y$ & $y$ & $y$ & $y$ & a & $y$ & $y$ & V & a & $y$ & $y$ \\
\hline
\end{tabular}

a NHA top-down

b MS main diagnosis + AP-DRG (somatic hospitals) or average daily rate (psychiatric clinics)

c MS average cost of psychiatric wards in somatic hospitals

d MS main diagnosis acute care + average daily rate for rehabilitation clinics

Statistic of Socio-Medical Institutions (Somed)

Helsana health insurance claims data

g Newlndex physicians' billing data

h Swiss Health Survey (SHS)

i MS information on admission via ambulance

j IMS Health market research data

$\checkmark$ Empty by definition

w Empty because no expenditures

$x$ Attribution of health services proportional to acute inpatient (for physicians) or physicians (for outpatient serv.)

$y$ Attribution of insurance administration costs proportional to overall health service spending 
Table 4 Decomposition of NHA expenditures by service and disease

\begin{tabular}{|c|c|c|c|c|c|c|c|c|c|c|c|c|c|c|c|c|c|c|c|c|c|c|}
\hline & \multirow{3}{*}{$\begin{array}{c}\text { National } \\
\text { Health } \\
\text { Accounts } \\
\text { total }\end{array}$} & & \multirow{3}{*}{ 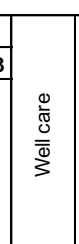 } & \multirow[b]{3}{*}{ 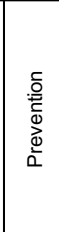 } \\
\hline & & \multicolumn{18}{|c|}{\begin{tabular}{|l|l|} 
Global Buden of Disease (GBD) classification \\
GBD 1 & GBD 2 - non-comm municable diseases
\end{tabular}} & \multirow{2}{*}{ 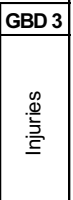 } & & \\
\hline & & 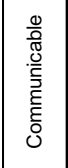 & 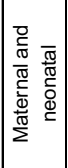 & 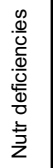 & 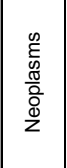 & 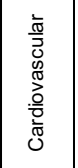 & 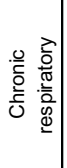 & 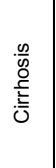 & 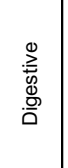 & 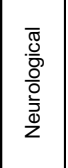 & 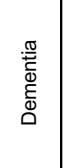 & 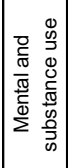 & 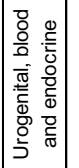 & 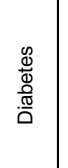 & 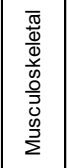 & 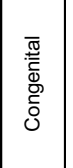 & $\frac{5}{\omega}$ & $\begin{array}{l}\Phi \\
\infty \\
\infty \\
\infty \\
\infty\end{array}$ & పूँ & & & \\
\hline Total expenditures & 64 '633 & 3'037 & 701 & 237 & $3^{\prime} 880$ & \begin{tabular}{|c|}
$10^{\prime} 084$ \\
\end{tabular} & $1^{\prime} 632$ & 196 & 3'018 & 1'309 & \begin{tabular}{|l|}
$1 ' 077$ \\
\end{tabular} & 6'861 & 4'387 & \begin{tabular}{l|l}
990 \\
\end{tabular} & $8^{\prime} 662$ & \begin{tabular}{|l|}
$2^{\prime} 968$ \\
\end{tabular} & \begin{tabular}{|l|}
$1 ' 199$ \\
\end{tabular} & \begin{tabular}{|l|}
1 \\
\end{tabular} & 3'965 & 5'216 & 1 1'526 & $22^{\prime} 592$ \\
\hline in percent of total & 100.0 & 4.7 & 1.1 & 0.4 & 6.0 & 15.6 & 2.5 & 0.3 & 4.7 & 2.0 & 1.7 & \begin{tabular}{|l|}
10.6 \\
\end{tabular} & \begin{tabular}{|r|}
6.8 \\
\end{tabular} & 1.5 & 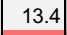 & 4.6 & 1.9 & 1.7 & \begin{tabular}{|r|}
6.1 \\
\end{tabular} & 8.1 & 2.4 & 4.0 \\
\hline rank from largest & & 8 & 19 & 20 & 7 & 1 & 12 & 21 & 9 & 14 & 17 & 3 & 5 & 18 & 2 & 10 & 15 & 16 & 6 & 4 & 13 & 11 \\
\hline \begin{tabular}{|l|} 
Inpatient care \\
\end{tabular} & $29^{\prime} 188$ & $1{ }^{\prime} 497$ & 563 & 169 & 2 '355 & 4'218 & 526 & 89 & \begin{tabular}{|l|}
$1 ' 643$ \\
\end{tabular} & 753 & 765 & \begin{tabular}{|l|}
$3^{\prime} 868$ \\
\end{tabular} & 1'957 & 214 & $3^{\prime} 727$ & 2'145 & 282 & 237 & 1 & 3'569 & 609 & \\
\hline Acute care & $16^{\prime} 847$ & 873 & 548 & 32 & $\mid 1^{\prime} 724$ & $2^{\prime} 314$ & 341 & 54 & 1'181 & 296 & 298 & 2'537 & 912 & 74 & 2'156 & 628 & 163 & 134 & 1 & 1'973 & 609 & \\
\hline Rehabilitation & $1^{\prime} 030$ & 25 & & 1 & 40 & 210 & 14 & 1 & 15 & 65 & 2 & 45 & 7 & 2 & 221 & 4 & 2 & 1 & & 374 & & \\
\hline Long-term nursing & $8^{\prime} 604$ & 600 & 15 & 136 & 591 & $1^{\prime} 695$ & 170 & 34 & 447 & 392 & 465 & 328 & \begin{tabular}{|l|}
1 \\
1
\end{tabular} 039 & 138 & 1'123 & 43 & 116 & 52 & 0 & $1^{\prime 2} 221$ & & \\
\hline Other long-term & $2 ' 707$ & & & & & & & & & & & 958 & & & 228 & 1'470 & & 50 & & & & \\
\hline Outpatient care ** & $21^{\prime} 501$ & 549 & 69 & 35 & 644 & \begin{tabular}{|l|}
$3^{\prime} 882$ \\
\end{tabular} & 539 & 60 & 679 & 361 & 187 & 1'560 & $1^{\prime} 314$ & 470 & $3^{\prime} 747$ & 248 & 548 & 501 & \begin{tabular}{|l|}
$3^{\prime} 921$ \\
\end{tabular} & 941 & 733 & 513 \\
\hline Phsysicians * & $9^{\prime} 277$ & 338 & 42 & 21 & 176 & $1^{\prime} 932$ & 335 & 37 & 418 & 223 & 114 & 540 & 809 & 254 & 2'011 & 126 & 338 & 308 & 30 & 317 & 519 & 387 \\
\hline Hospitals & $5^{\prime} 380$ & 110 & 14 & 7 & 398 & $1^{\prime} 272$ & 104 & 12 & 136 & 72 & 38 & 652 & 263 & 146 & 1'114 & 41 & 110 & 100 & 10 & 489 & 168 & 126 \\
\hline Dental & $3^{\prime} 873$ & & & & & & & & & & & & & & & & & & 3'873 & & & \\
\hline Physiotherapy & 811 & 30 & 4 & 2 & 16 & 70 & 30 & 3 & 37 & 20 & 10 & 58 & 71 & 22 & 196 & 22 & 30 & 27 & 3 & 114 & 46 & \\
\hline Psycho & 220 & & & & & & & & & & & 220 & & & & & & & & & & \\
\hline & $1 ' 734$ & 64 & 8 & 4 & 54 & 568 & 64 & 7 & 80 & 42 & 22 & 72 & 154 & 43 & 414 & 1 & 64 & 59 & 6 & 9 & & \\
\hline Other outpatient & 208 & 7 & 1 & 0 & 0 & 40 & 6 & 1 & 9 & 5 & 2 & 19 & 17 & 5 & 11 & 58 & 7 & 6 & & 12 & & \\
\hline \begin{tabular}{|l|} 
Other services \\
\end{tabular} & 2'102 & 152 & 14 & 5 & 80 & 434 & 66 & 8 & 118 & 60 & 25 & 94 & 235 & 67 & 218 & 9 & 44 & 29 & 7 & 366 & 24 & 44 \\
\hline Laboratory analysis & 997 & 64 & 6 & 2 & 46 & 221 & 42 & 4 & 50 & 25 & 12 & 39 & 181 & 55 & 139 & 1 & 32 & 15 & 6 & 4 & 13 & 36 \\
\hline & 187 & 7 & 1 & 0 & 4 & 40 & 7 & 1 & 9 & 5 & 2 & 11 & 17 & 5 & 42 & 3 & 7 & 6 & 1 & 2 & 11 & 8 \\
\hline Transport and rescue & 918 & 80 & 8 & 3 & 30 & 173 & 17 & 4 & 59 & 31 & 11 & 44 & 37 & 6 & 37 & 5 & 5 & 7 & 0 & 359 & 1 & \\
\hline Sales of health goods & $7 ' 360$ & 690 & 21 & 17 & 610 & \begin{tabular}{|l|}
$1 ' 056$ \\
\end{tabular} & 422 & 29 & 430 & 70 & 48 & $11^{\prime} 002$ & 665 & 190 & 547 & 263 & 266 & 275 & 36 & 164 & 85 & 473 \\
\hline Drugs & 6'079 & 650 & 16 & 14 & 590 & 827 & 382 & 24 & 381 & 44 & 34 & 938 & 570 & 160 & 309 & 40 & 226 & 239 & 32 & 104 & 24 & 473 \\
\hline Medical devices & $1^{\prime} 281$ & 40 & 5 & 3 & 21 & 228 & 40 & 4 & 49 & 26 & 14 & 64 & 96 & 30 & 238 & 223 & 40 & 36 & 3 & 60 & 61 & \\
\hline Prevention & $1^{\prime} 434$ & & & & & & & & & & & & & & & & & & & & & $1^{\prime} 434$ \\
\hline Administration & $33^{\prime} 048$ & 149 & 34 & 12 & 190 & 494 & 80 & 10 & 148 & 64 & 53 & 336 & 215 & 48 & 424 & 302 & 59 & 54 & & 177 & 75 & 127 \\
\hline
\end{tabular}

Decomposition of 2011 Swiss National Health Accounts (NHA) in Swiss francs by disease. Empty cells hold no expenditures because the type of disease does not fit with the type of service or because no expenditures were assigned to these cells

* $0.63 \%$ of spending for outpatient physicians' spending was attributed proportionally to inpatient care spending

**The following shares of spending for services and health goods were assigned proportionally to outpatients physicians' spending: $17.87 \%$ of outpatient care without physicians, $55.33 \%$ of other outpatient services and $14.08 \%$ of health goods

of inpatient care ranged from $0.0 \%$ in oral disorders to $84.4 \%$ in maternal and neonatal disorders. Among the highexpenditure disease groups, the proportion of inpatient care was particularly high for injuries $(70.8 \%)$, neoplasms (63.8\%) and mental disorders (59.3\%). Outpatient care was particularly important in the treatment of oral disorders (99.1\%), diabetes $(57.1 \%)$, well care $(52.2 \%)$, skin diseases (51.9\%), and sense organ diseases (50.8\%). The diseases with the highest proportion of outpatient drug and medical device expenditures were communicable diseases (23.9\%), chronic respiratory diseases (27.2\%), sense organ diseases (26.4\%), skin diseases (23.3\%) and diabetes (20.2\%).

We also assessed the correlation between disease and service-specific healthcare expenditures and the two dimensions of the health burden of diseases: years lived with disability (YLD) and years of life lost due to premature mortality (YLL). Both YLDs and YLLs for Switzerland in the year 2010 were available from the GBD Study [23]. The correlation between spending and burden of disease was substantially stronger with YLDs (coefficient of 0.591) than with YLLs (coefficient of 0.404). The strongest correlation was between spending and DALYs, which equate to the sum of YLDs and YLLs (coefficient of 0.747).

\section{Discussion}

Our study is the first to decompose Swiss healthcare spending by major disease. We thus added a fifth dimension to Swiss NHA in addition to the four dimensions already provided by the FOS [4] (financing agent, financing regimen, type of service providers and type of service). The results showed that non-communicable diseases dominated healthcare spending in 2011 accounting for $79.4 \%$ of total spending. The five most costly non-communicable major diseases (cardiovascular, musculoskeletal, mental, urogenital and hematological, oral) accounted for over $50 \%$ of spending. Chronic diseases, such as musculoskeletal and mental disorders, turned out to be more important drivers of healthcare spending than diseases associated with premature mortality such as neoplasms.

The methodological approach combined a top-down and a bottom-up approach, which allowed for assigning the 


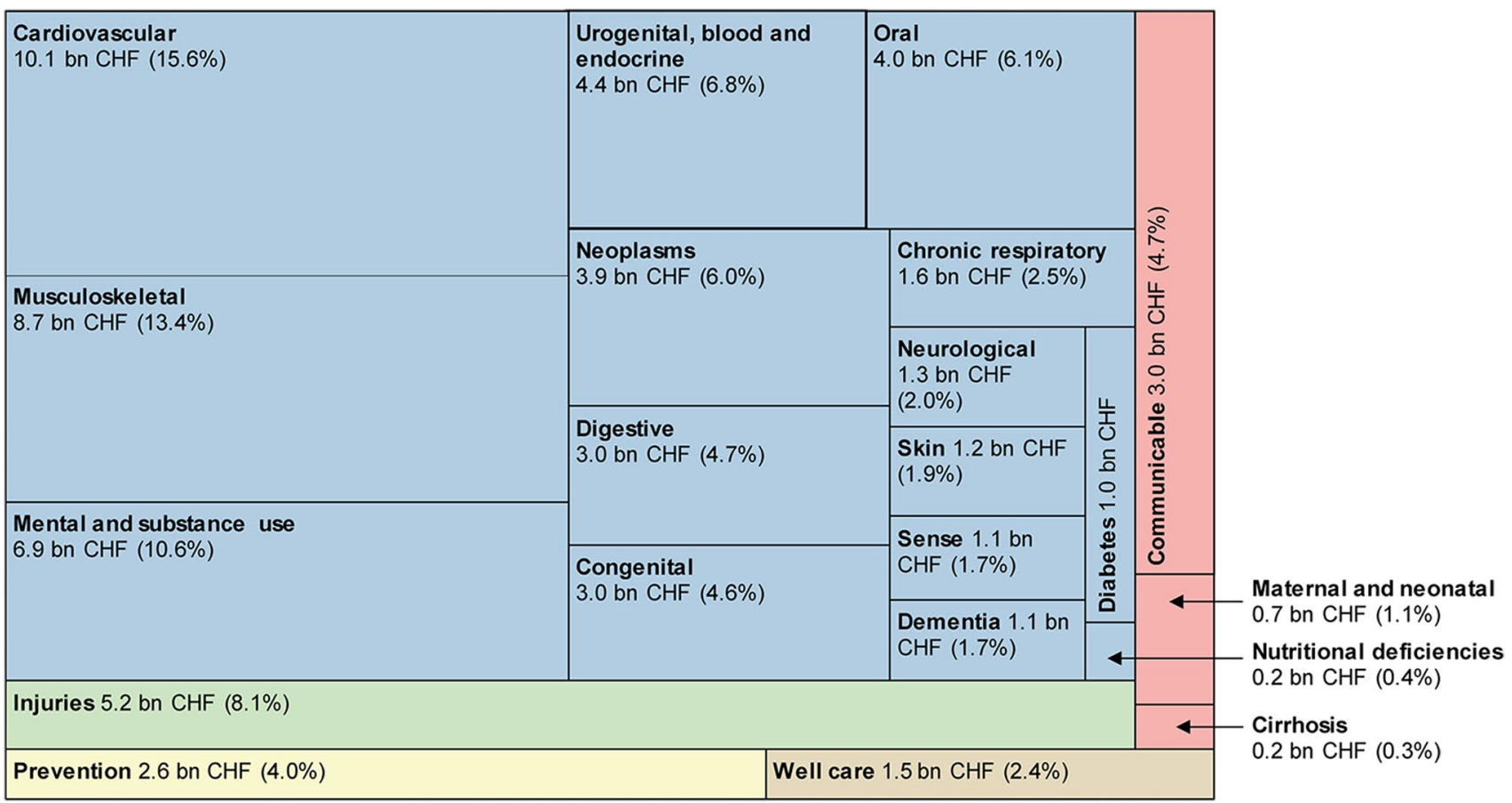

Fig. 1 NHA expenditures by disease group and disease. The area of the boxes represents the share of a disease or disease group in total Swiss National Health Accounts expenditures in 2011. Exchange rates of Swiss franc (CHF) in 2011: $0.811 \mathrm{EUR} / \mathrm{CHF}, 1.127 \mathrm{USD} /$ CHF [21]. Non-communicable diseases (in blue) account for $79.4 \%$ of total spending
Fig. 2 Share of main service categories in disease expenditures. Shares of expenditures (net of health insurance administration costs) in disease expenditures. Diseases are ordered by the share of total inpatient spending (from highest to lowest)

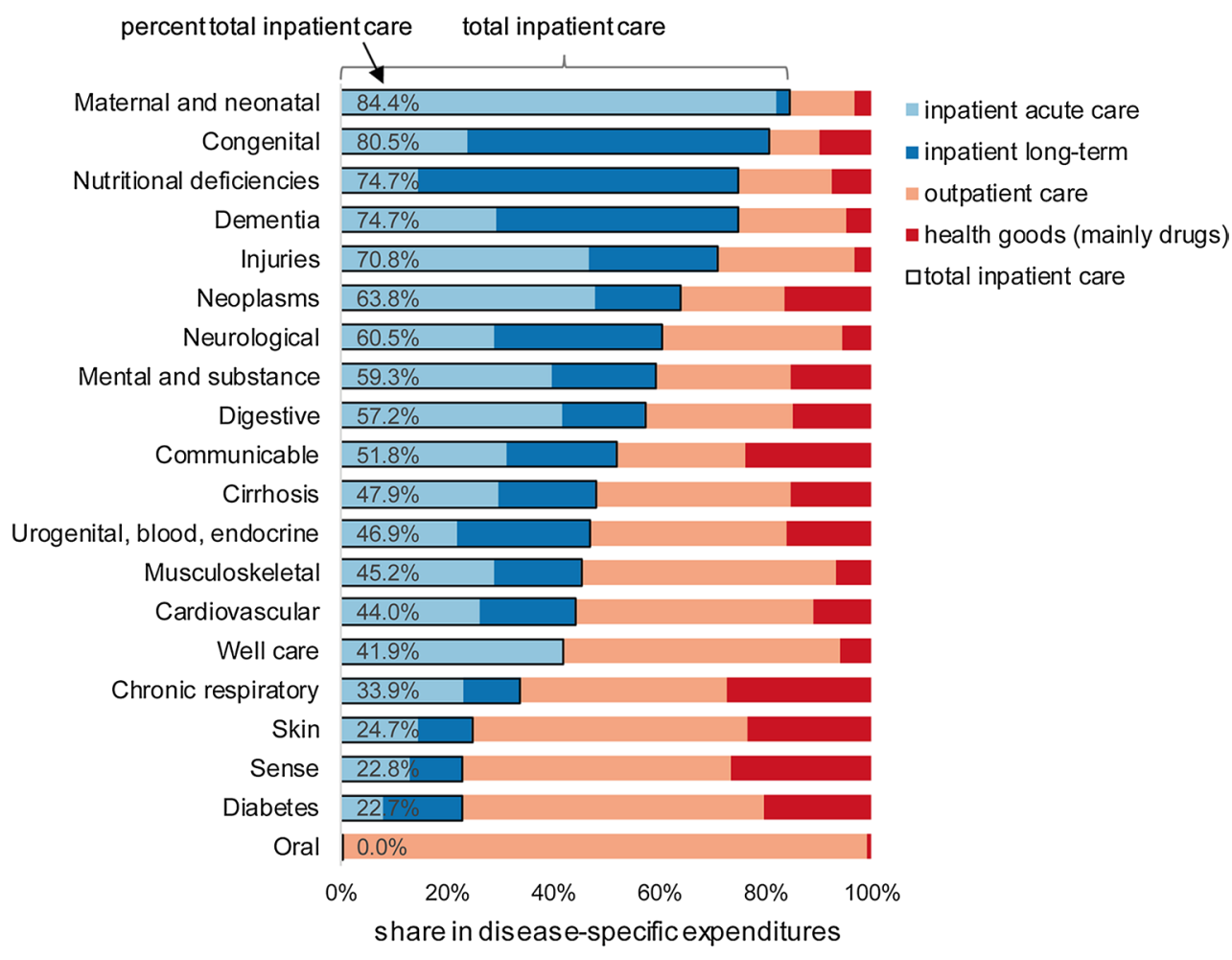


majority of service-specific expenditures to different disease categories. The accuracy of the assignment varied between service categories and largely depended on the availability of high-quality data. Diseases were identified by diagnostic codes of treatment episodes (e.g., ICD-10 codes in hospital registry data), by the type and specialization of service providers (e.g., psychiatric hospitals, dentist, oncologist, rheumatologist), and by the approved indications of drugs. In addition, attribution of spending to diseases was facilitated by two cause-specific social insurance schemes allowing for identifying spending for injuries (accident insurance) and congenital diseases (disability insurance) in NHA.

We are rather confident about the accuracy of our expenditure attribution for service-disease cells with high-quality information for disease identification. This confidence applies to most inpatient care cells and to outpatient-care cells representing high prevalence disease categories and major healthcare service categories. However, accuracy is poorer for low prevalence diseases and less central healthcare service categories, such as laboratory tests. Due to a lack of data, cirrhosis, nutritional deficiencies, physiotherapy, outpatient nursing and radiology cells had to be filled according to the cells' relative importance at the level of expenditures for outpatient physician services. The impact on the overall accuracy of the expenditure mapping of diseases is nonetheless limited, as the subsequent assigned spending represented only $5.5 \%$ of total spending.

Our results can be interpreted as the direct expenditures for the treatment of a disease. The expenditures for the treatment of the comorbidities affecting a patient are thus only partially included in the expenditures for the primary disease. This is particularly true for expenditures assigned based on the type of financing regimen, the type of service, the specialization of the physician, or the ATC-code (for drug expenditures), as the underlying data sources allow for a precise separation of spending by disease. However, it is likely that some spending on comorbidities was also included in the inpatient acute care expenditures assigned to specific diseases, although the main diagnosis assigning expenditures to diseases was the diagnosis responsible for most of the costs. Spending on some comorbidities may also be included in the expenditures assigned with regression models, as these models could not control for all possible comorbidities.

Furthermore, the expenditures for the direct treatment of a disease differ from all the expenditures triggered by a disease. The case of diabetes may be useful in illustrating this point, as diabetes is an important risk factor for cardiovascular diseases, sense organ diseases and many other diseases. From an etiological perspective the expenditures for diabetes are likely to be much higher than our observed $1.5 \%$ of total healthcare expenditures, as a substantial part of the diseases induced by diabetes would be reallocated to diabetes. The case of osteoporosis (a musculoskeletal disorder) and falls (an injury) may further illustrate this point. Falls by elderly patients affected by osteoporosis often require intensive inpatient treatment. The corresponding healthcare spending would appear among the injuries in our classification, while they would be assigned at least partially to musculoskeletal disorders from an etiological perspective.

Our results are in line with the decomposition of US personal healthcare spending in Dieleman et al. [11] and in Roehrig et al. [10]. The ranking and scale of spending for major diseases determined in these studies are similar to our results for Switzerland. Conversely, our results diverge somewhat from those of Dunn and coauthors $[15,16]$, based on the Health Care Satellite Account developed by the US Bureau of Economic Analysis. These differences are in part due to differences in the disease classification. The second most important spending category in Dunn et al. [15] is for example "Routine care, signs and symptoms" (12.4\% of spending), which does not appear in the GBD classification applied Dieleman et al. [11] and our study. A comparison with the results of Heijink and coauthors [6] for Australia, Canada, France, Germany and the Netherlands and with more recent findings for a number of OECD countries [8] shows larger differences with our results. However, these differences may be largely attributable to the methodological limits of the spending decompositions for these countries, such as the allocation of only a part of total spending to diseases [6].

The results of our study may be useful to researchers and health policy makers in several ways: first, better knowledge of the distribution of overall healthcare spending across diseases may contribute to more efficient priority setting for health policies and budgetary planning. If life expectancy is continuing to increase and medical progress transforms more lethal diseases into chronic ones, then spending for high prevalence chronic diseases is expected to grow disproportionally. Second, the results provide an overall reference for single cost-of-illness studies. This reference may be useful to verify the plausibility of single cost-of-illness studies, and thus helps to prevent misallocations of resources because of the risk of overestimation and double counting in single cost-of-illness studies [2]. Third, our study illustrates how healthcare spending can be assigned to single diseases when no diagnostic information is available. This may be useful for the decomposition of healthcare spending in countries with limited diagnostic information in expenditure or service use data. Fourth, our results may serve as an input for studies assessing the healthcare costs attributable to behavioral or environmental risk factors such as smoking or air pollution. Such studies typically assess the fractions of disease prevalence or incidence attributable to a risk factor of interest. These attributable fractions are then multiplied 
with the healthcare spending for single diseases to calculate the spending attributable to the risk factor.

Our study has a number of limitations. First, our analytical options were restricted by the limited access to health insurance claims data. Broader access to such data would have allowed for a substantially better attribution of expenditures to service-disease cells related to minor outpatient services and relatively low disease prevalence. However, these restrictions were mainly due to limited resources available and could be overcome in future estimations. Second, due to a lack of suitable data, the expenditures related to a number of service-disease cells had to be attributed proportionally to previously assigned cells. However, the consequences on overall results are likely to be limited as only $5.5 \%$ of the total spending was assigned proportionally. Third, the accuracy of the attribution of expenditures differs substantially between disease and service categories. While accuracy is higher for inpatient acute care and outpatient physician and hospital services, it is lower for long-term inpatient care and minor outpatient services. Thus, there is much room for the use of better data and the development of improved methods for the cost attribution. Fourth, with our approach, the granularity of the decomposition is often limited to a decomposition by main disease groups. The expenditures for the disease category neoplasms can, for example, not be decomposed by different types of neoplasms based on the physicians' specialization or types of drugs, as oncologists may treat patients affected by different types of neoplasms and many drugs are used to treat different forms of neoplasms. More detailed diagnostic coding would thus be required for decomposition with higher granularity.

Further research may include the decomposition of disease-specific spending by age, gender and type of payer. Better access to health insurance claims data may also allow for higher granularity in disease decomposition, particularly for the high-expenditure cardiovascular diseases, musculoskeletal disorders and mental and substance use disorders. Our study may also serve as a starting point for further research into the drivers of the constant growth of healthcare spending. To this end, disease-specific spending must be assessed in different years. The changes in spending over time could then be decomposed into changes in the age structure and size of the population, in the prevalence of diseases, in the access-to-care restrictions, in the intensity and type of care, and in the prices of healthcare services and products.

Acknowledgments We thank the Swiss Federal Office of Public Health for funding a first version of this study and the Swiss Federal Office of Statistics for providing us with a large part of the data used. We thank Klaus Eichler for his advice on mapping IDC-codes to diseases and Michael Stucki and Maria-Eleni Syleouni for their research assistance. We are particularly grateful to Joseph Dieleman of the Institute of Health Metrics and Evaluation of the University of Washington for hosting the corresponding author during the preparation of the manuscript and for his much appreciated feedback on a first version of the paper.

\section{Compliance with ethical standards}

Conflict of interest The authors declare no conflicts of interest. Mark Pletscher is an employee of Hoffmann-La Roche Ltd. At the time of the study, Mark Pletscher was working for the Winterthur Institute of Health Economics, Zurich University of Applied Sciences.

Open Access This article is distributed under the terms of the Creative Commons Attribution 4.0 International License (http://creativeco mmons.org/licenses/by/4.0/), which permits unrestricted use, distribution, and reproduction in any medium, provided you give appropriate credit to the original author(s) and the source, provide a link to the Creative Commons license, and indicate if changes were made.

\section{References}

1. Onukwugha, E., McRae, J., Kravetz, A., Varga, S., Khairnar, R., Mullins, C.D.: Cost-of-illness studies: an updated review of current methods. PharmacoEconomics 34(1), 43-58 (2016). https:// doi.org/10.1007/s40273-015-0325-4

2. Bloom, B.S., Bruno, D.J., Maman, D.Y., Jayadevappa, R.: Usefulness of US cost-of-illness studies in healthcare decision making. PharmacoEconomics 19(2), 207-213 (2001). https://doi. org/10.2165/00019053-200119020-00007

3. Rosen, A.B., Cutler, D.M.: Challenges in building disease-based national health accounts. Med. Care 47(7 Suppl 1), S7-S13 (2009). https://doi.org/10.1097/MLR.0b013e3181a23e12

4. Swiss Federal Office of Statistics: Kosten und Finanzierung des Gesundheitswesens (costs and financing of healthcare). Swiss Federal Office of Statistics (2014)

5. OECD, Eurostat, WHO: A System of Health Accounts. OECD Publishing (2011)

6. Heijink, R., Noethen, M., Renaud, T., Koopmanschap, M., Polder, J.: Cost of illness: an international comparison: Australia, Canada, France, Germany and The Netherlands. Health Pol 88(1), 49-61 (2008). https://doi.org/10.1016/j.healthpol.2008.02.012

7. OECD: Health at a Glance 2015: OECD Indicators. OECD Publishing, Paris (2015)

8. OECD: Focus on Health Spending - Expenditure by disease, age and gender. OECD, Paris (2016)

9. Dieleman, J., Campbell, M., Chapin, A., et al.: Evolution and patterns of global health financing 1995-2014: development assistance for health, and government, prepaid private, and outof-pocket health spending in 184 countries. Lancet 389(10083), 1981-2004 (2017). https://doi.org/10.1016/S0140-6736(17)30874 $-7$

10. Roehrig, C., Miller, G., Lake, C., Bryant, J.: National health spending by medical condition, 1996-2005. Health Aff (Millwood) 28(2), w358-w367 (2009). https://doi.org/10.1377/hltha ff.28.2.w358

11. Dieleman, J.L., Baral, R., Birger, M., et al.: US spending on personal health care and public health, 1996-2013. JAMA 316(24), 2627-2646 (2016). https://doi.org/10.1001/jama.2016.16885

12. Roehrig, C.S., Rousseau, D.M.: The growth in cost per case explains far more of US health spending increases than rising disease prevalence. Health Aff (Millwood) 30(9), 1657-1663 (2011). https://doi.org/10.1377/hlthaff.2010.0644

13. Thorpe KE, Florence CS, Joski P (2004) Which medical conditions account for the rise in health care spending? Health Aff 
(Millwood) Suppl Web Exclusives, W4-437-445. https://doi. org/10.1377/hlthaff.w4.437

14. Thorpe, K.E.: Treated disease prevalence and spending per treated case drove most of the growth in health care spending In 19872009. Health Aff. 32(5), 851-858 (2013). https://doi.org/10.1377/ hlthaff.2012.0391

15. Dunn, A., Rittmueller, L., Whitmire, B.: Health care spending slowdown from 2000 to 2010 was driven by lower growth in cost per case, according to a new data source. Health Aff (Millwood) 35(1), 132-140 (2016). https://doi.org/10.1377/hlthaff.2015.1109

16. Dunn A, Rittmueller L, Whitmire B (2015) Introducing the New BEA Health Care Satellite Account. Survey of Current Business 95(1)

17. Dieleman, J., Squires, E., Bui, A.L., et al.: Factors associated with increases in us health care spending, 1996-2013. JAMA 318(17), 1668-1678 (2017). https://doi.org/10.1001/jama.2017.15927

18. Bui, A.L., Lavado, R.F., Johnson, E.K., et al.: National health accounts data from 1996 to 2010: a systematic review. Bull. World Health Organ. 93, 566-576 (2015). https://doi.org/10.2471/ BLT.14.145235

19. De Pietro, C., Camenzind, P., Sturny, I., et al.: Switzerland: health system review. Health Syst Transit 17(4), 1-288 (2015)

20. OECD: OECD Health Statistics. OECD. http://stats.oecd.org/ index.aspx ?DataSetCode=HEALTH_STAT (2017). Accessed July 22, 2017

21. Swiss National Bank: Foreign exchange rates - Annual average. Swiss National Bank

22. WHO: Global Health Observatory data repository-life expectancy. World Health Organization. http://apps.who.int/gho/data/ node.main.688 (2017)

23. Murray, C.J.L., Vos, T., Lozano, R., et al.: Disability-adjusted life years (DALYs) for 291 diseases and injuries in 21 regions,
1990-2010: a systematic analysis for the global burden of disease study 2010. Lancet 380(9859), 2197-2223 (2012)

24. Larg, A., Moss, J.R.: Cost-of-illness studies: a guide to critical evaluation. Pharmacoeconomics 29(8), 653-671 (2011)

25. Swiss Federal Office of Statistics: Kodierungshandbuch. Der offizielle Leitfaden der Kodierregeln (coding manual for the Medical Statistic of Hospitals). Swiss Federal Office of Statistics (2009)

26. Swiss Federal Office of Statistics: Diagnosebezogene Fallkosten 2011 (diagnosis related case costs 2011). Bundesamt für Statistik, Neuchâtel (2013)

27. Huber, C.A., Szucs, T.D., Rapold, R., Reich, O.: Identifying patients with chronic conditions using pharmacy data in Switzerland: an updated mapping approach to the classification of medications. BMC Publ. Health 13(1), 1030 (2013). https://doi. org/10.1186/1471-2458-13-1030

28. Swiss Federal Office of Statistics: Swiss Health Survey. Swiss Federal Office of Statistics, Neuchâtel (2012)

29. IHME: GBD Compare Data Visualization. Institute for Health Metrics and Evaluation, University of Washington. https://vizhu b.healthdata.org/gbd-compare/(2016). Accessed May 5, 2017

30. Kelley, A.S., McGarry, K., Gorges, R., Skinner, J.S.: The burden of health care costs in the last 5 years of life. Ann. Intern. Med. 163(10), 729-736 (2015). https://doi.org/10.7326/M15-0381

31. Rechel, B., Doyle, Y., Grundy, E., McKee, M.: How can health systems respond to population ageing? World Health Organization, on behalf of the European Observatory on Health Systems and Policies (2009)

32. National Institute on Aging, WHO: Global Health and Aging. National Institute of Health (2011) 